\title{
Solutions to operate transmission and distribution gas networks
}

\author{
Sorin Neacsu', and Cristian Eparu ${ }^{2, *}$ \\ ${ }^{1}$ UPG Ploiesti, DETH Department, 39 Bucharest Blvd, Ploiesti, România, \\ ${ }^{2}$ NetGas R\&D, 28 Bucharest Blvd, Ploiesti, România
}

\begin{abstract}
In order to respect the actual and future regulations, besides SCADA, there is a need for further modern operating solutions for the transmission and distribution gas network. The paper presents the newest operating principles and modern software solutions that represent a considerable help to operate the transmission and distribution gas networks.
\end{abstract}

\section{Introduction. AdmoduNet simulator, real time operating solution for gas transport and distribution systems}

The modernization of natural gas transport and distribution systems has necessitated the introduction of SCADA systems. These are offering at each moment a snapshot of the situation of the transport system. The information is discontinuous because of the large area of the transport and distribution network and the big number of pipes. The number of points where data are acquired are limited due to costs.

The transport and distribution process is a continuous process and in order to get a precise image of the parameters at any moment in time it is necessary that, besides SCADA systems, a dynamic simulator calibrated for the respective network to be in place [1]. This simulator can complete the picture of parameters in the entire transport or distribution network based on the real data received. In this way, the parameters that are to be measured, like line pack for pipes or the entire network [2], spatial and time continuous distribution of transport parameters (pressure, flow rate, temperature) and also gas quality (composition, calorific value) can be determined [3].

Having a nonstationary simulator calibrated on a transport or distribution network allows making simulations for preparing the next day plan of transport (base plan). The simulator can also be used to predict the flow in the system for bigger time intervals: days, weeks, months and years. Another important function is the possibility of coming up, very quickly, with a solution in case of accidents that can deviate from the normal functioning of the system (base plan).

NetGas R\&D has developed the AdmoduNet simulator for gas transport and distribution and oil and gas gathering systems. The simulator contains calculation engines for monophase (gas, liquid) and multiphase systems.

The simulator concentrates all calculation effort on servers and has a multiuser type web interface so that it can be used by multiple departments in a company, even from mobile devices.

\footnotetext{
${ }^{*}$ Corresponding author: sneacsu.ph@gmail.com
} 
AdmoduNet has performant proxy models for all regulation points in the network (compressors, control valves, slug catchers) that allow simulating any desired command.

\section{Necessary data for a base plan}

The transport program is dynamic due to the gas capacities provided by the gas market. In order to define these quantities, AdmoduNet has a business module through which the equilibration of demand and offer from the market is possible. It should be mentioned here that the connections between the gas suppliers and the customers form an entire different transport network called commercial network. The business module makes the daily equilibration of this commercial network (fig. 1), converts commercial agreements into pointto-point Demand/Supply transactional relationship configurations and enables synoptic Business Rules visualization, converts Business Rules and current supply/demand constraints into operational conditions for process simulation

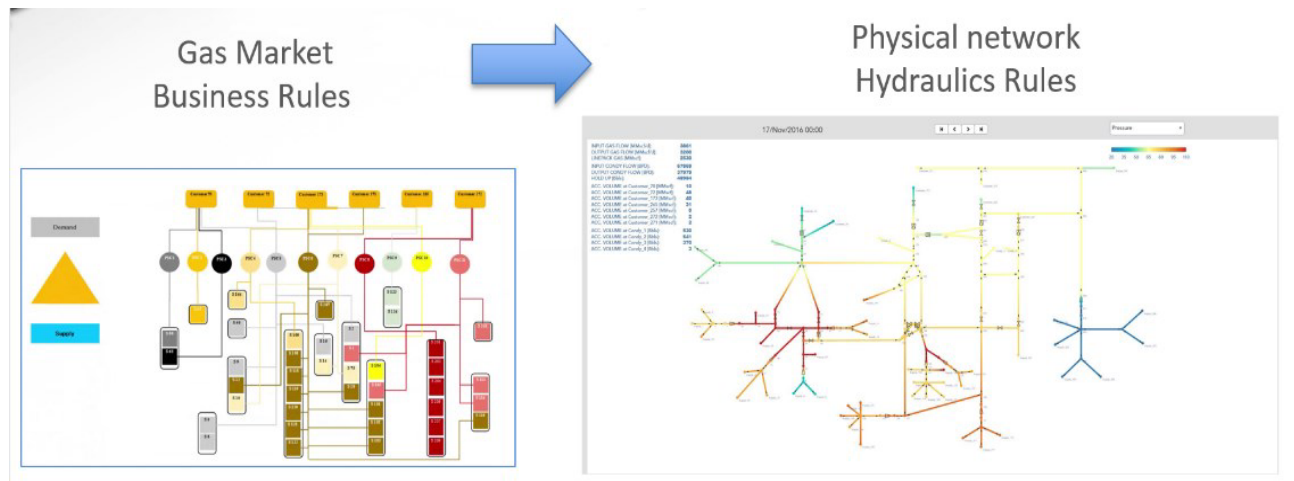

Fig. 1. Differences between commercial and physical network

The consumption volumes used for network entry and exit points are global values for 24 hours that came from the gas market. For these values to be properly used in dynamic simulations, there are modeled with hourly profiles defined for each consumption point from historical data [4] (fig. 2).
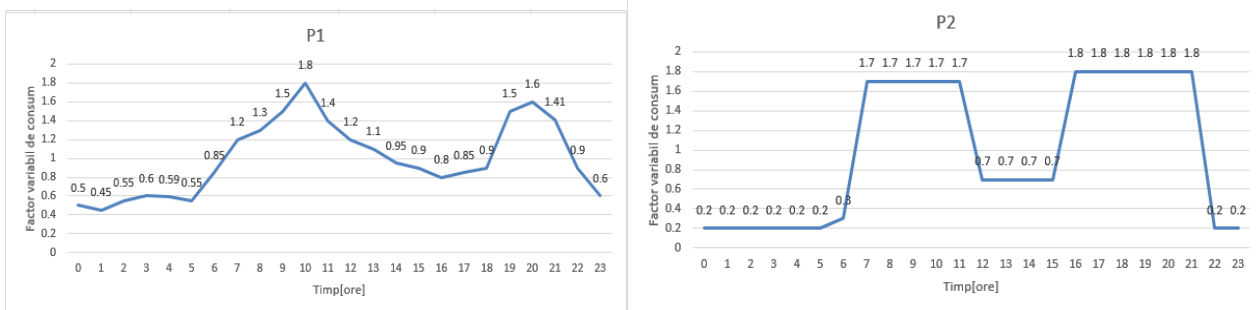

Fig. 2. Hourly consumption profiles for a locality and an industrial consumer

A performant profiling module that has the ability to define a consumption profile based on temperature, day of the week and other major influences, is used in order to determine the small clients' (non-monitored) consumptions (fig. 3). This module is used for modelling the consumption behavior of an area in order to get reliable prognosis for the gas volumes needed. 

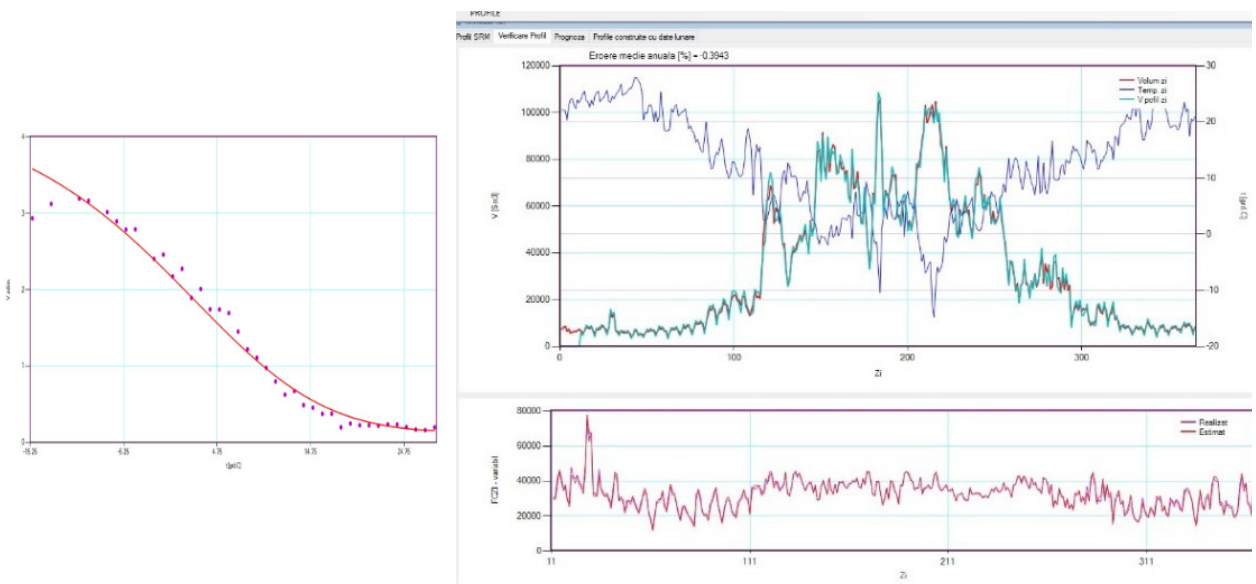

Fig. 3. Profile use and gas volume nomination

\section{Dynamic simulations with AdmoduNet}

Once the transport system load is defined, by establishing the gas values at the entry and exit points, necessary simulation can be made starting from an initial state of the transport system. Figure 4 presents a graphic representation of the results of a simulation.

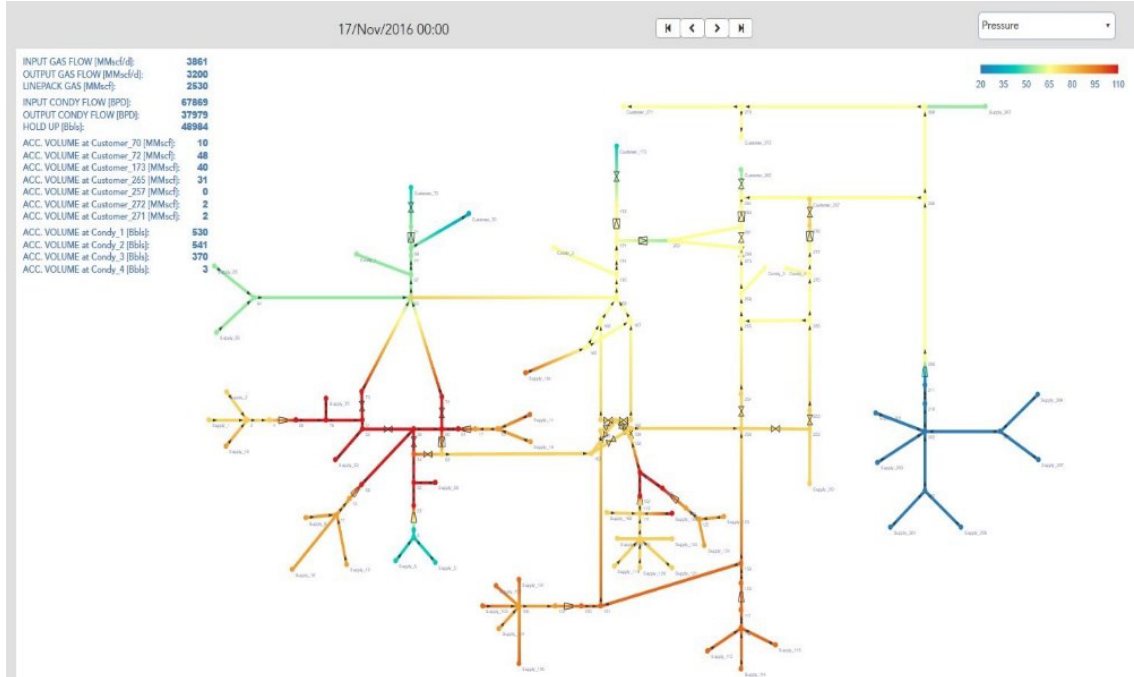

Fig 4. View of pressure variation in the network

The parameter chosen to be presented in this case is pressure. The coloring of the pipes is based on a color code. In the left upper part of the picture global values for the network are presented for a specific moment in time. We can see the evolution in time for any defined parameter by clicking the middle buttons.

The software allows different types of results visualization directly from the main tab.

In figure 5 one can see the possibility of graphically viewing different parameters and gas composition variations in space (every node of the network) and time. 


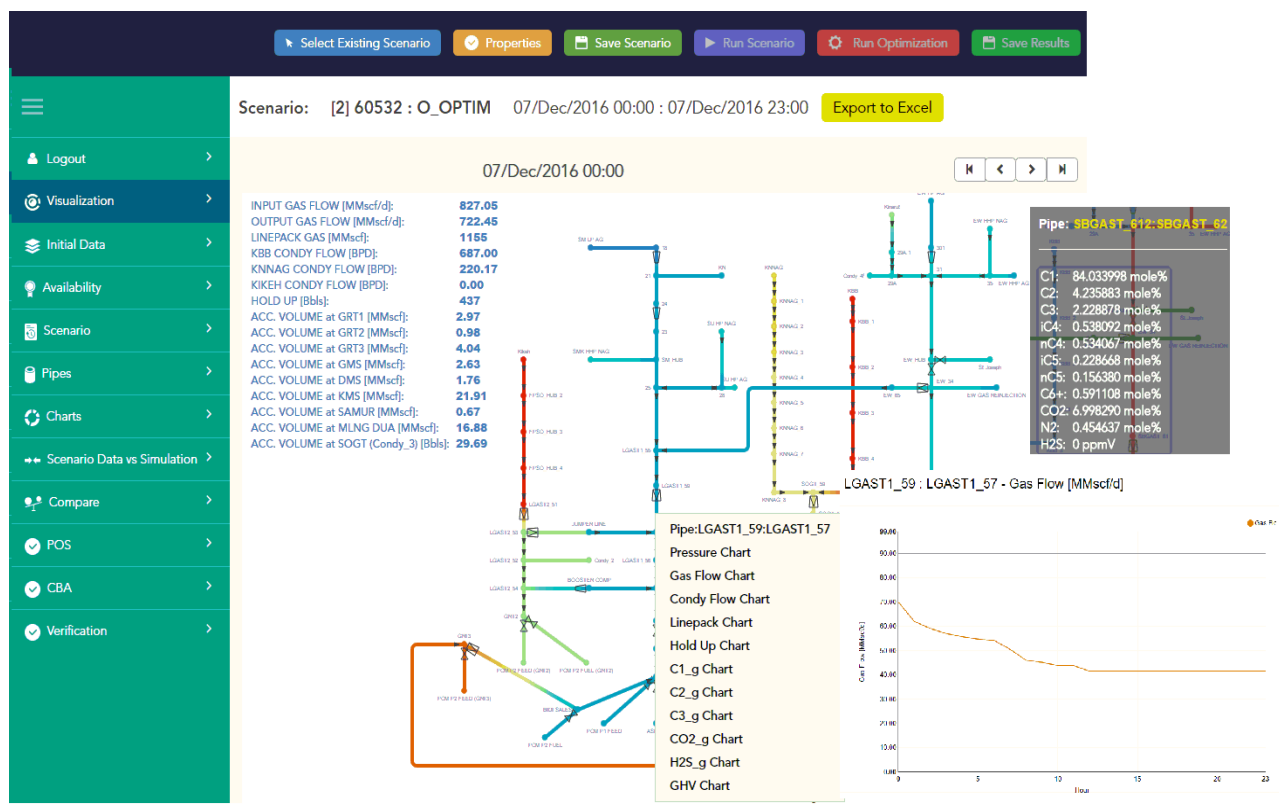

Fig 5. Results visualization

Figure 6 presents different possibilities of graphic representation for gas volume delivered to the client, variation of pressure on a path that connects an entry point to an exit point and priority of supply, where we can see from which supply point came the gas delivered to a customer. This last calculation is the base for cost business analysis.

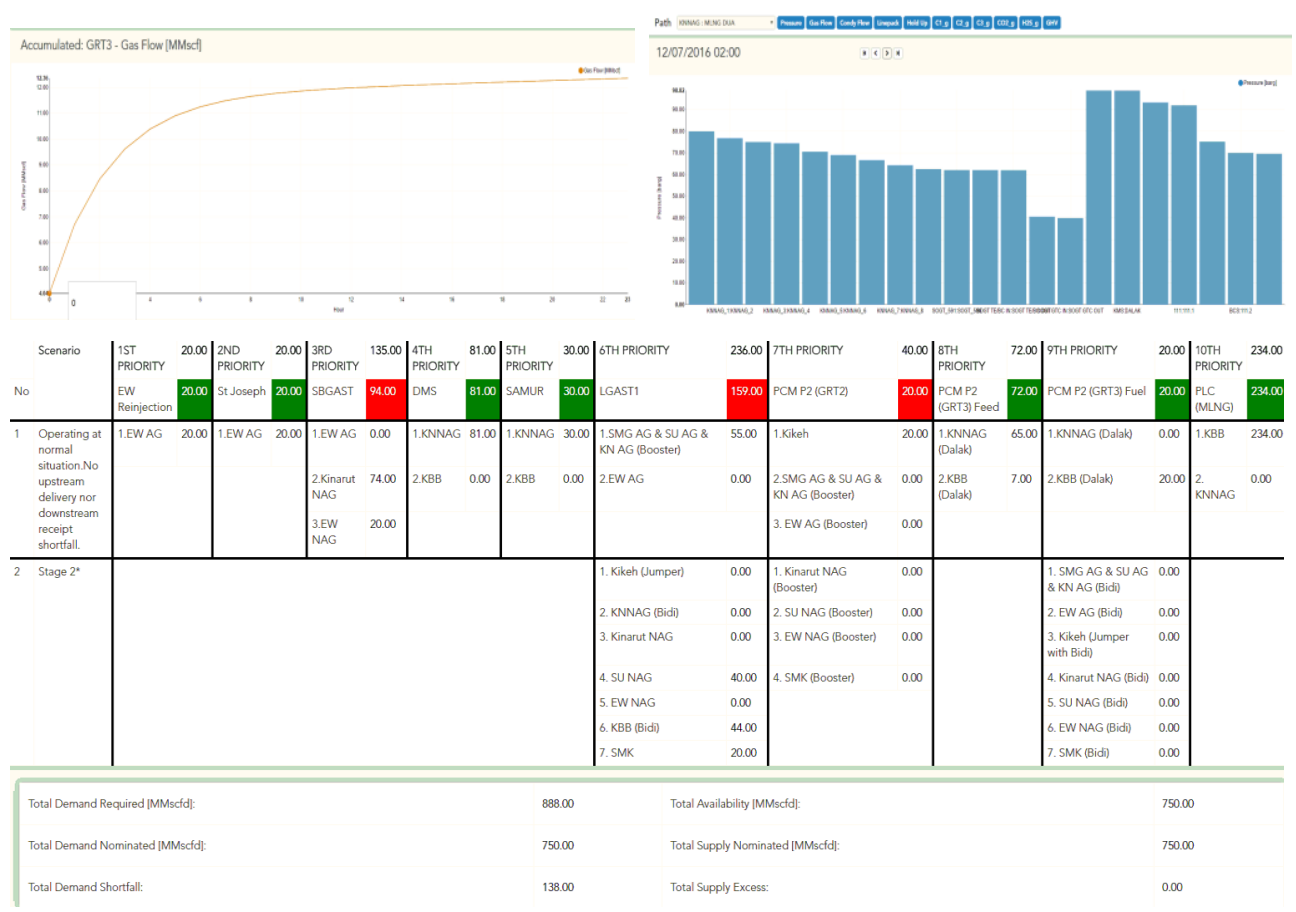

Fig. 6. Results analysis and visualization 


\section{Simulation types available with AdmoduNet}

Forward simulation starts from gas volumes at supply points and determine the quantity and quality of gas that will be delivered to the customers, taking into consideration the restriction and parameters for control elements [5].

Reverse simulation provides the best operating scenario starting from the gas delivery target on volumes and quality and determines the platforms and network set-points of control elements and operational conditions. Because multiple solutions can exist, this type of simulation is used together with an optimization module that can be configured based on the clients' needs.

Auto-simulation (Monitoring) is used in the dispatch center by the operators; it is a very important predictive application that will give the operators the necessary comfort to make decisions in a real time, solving thus future problems that can appear in the system. The monitoring application automatically simulates the transport process every hour for the remaining time ahead in the monitor time horizon, compares the results simulated with the initial base plan and also identifies the deviation from plan in the remaining time ahead.

In figure 7 one can see a predictive exception based on monitoring which shows what will happen in the future as an exception to your plan. What is shown here is a line which is the plan line/base, a line that was created the day before and then, every hour, it shows you what will happen in the next hours, more precisely what may deviate from that plan. It is a predictive exception surveillance, different from any other surveillance known so far, that is actually showing a deviation only after the detection of a problem.
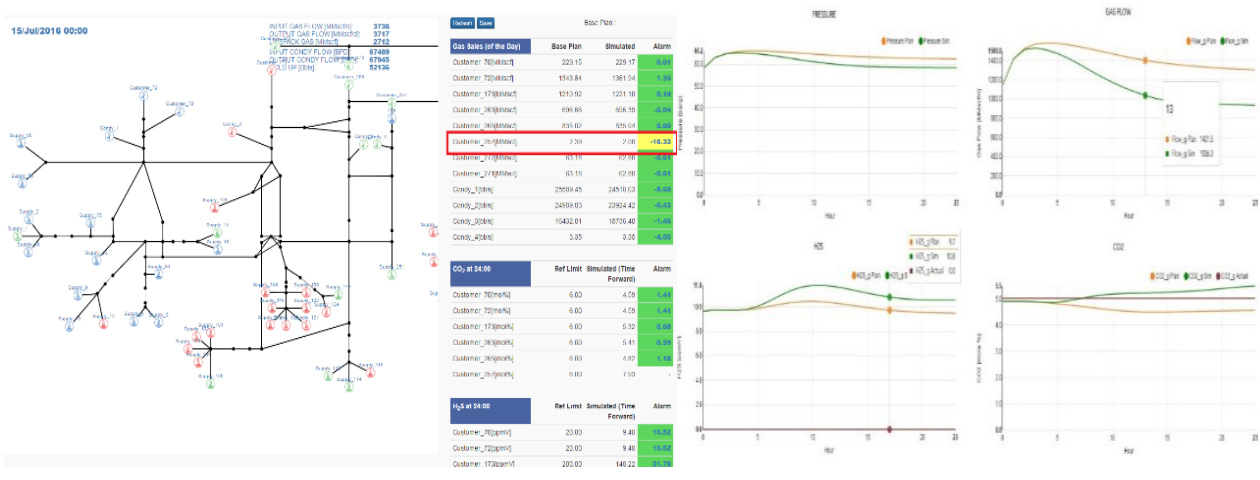

Fig. 7. Monitoring dashboard and graphs

By fulfilling the objective of simulation (supplied and delivered quantities, gas quality, pressure regime in the network) we obtain the operation mode, represented by the totality of commands needed to be executed and the moment in time when these commands must be executed for all the control elements in the network (control valves, compressors).

In the case of gas distribution system, where only large consumers (B4-B6) are monitored, NetGas R\&D prepared a similar solution with AdmoduNet that can be used to make the equilibration of the distribution network every 24 hours, based on data from a limited number of control points in the network.

\section{Conclusions}

Transport and distribution systems are spread on large areas and have many pipelines. SCADA systems give information from a limited number of points in the network. In order to determine the flow parameters in the entire network, a dynamic simulator has to be used, 
a simulator that continuously determines the parameters values in the whole network starting from the monitoring data.

From the dynamic simulation, a very important measure for the equilibration process and its variation is obtained.

The use of a performant dynamic simulator allows the operation process for the next time interval to be defined.

In case of various incidents, by using the optimization module, low-priced operational solutions can be easily and promptly found.

Certification of correct solution results of AdmoduNet simulator presented in this paper is represented by Petronas using the gas and condensate gathering system from offshore platforms.

\section{References}

1. S. Neacșu, C. Eparu, Proceedings of the 21st International Business Information Management Association (IBIMA) Conference, (Vienna, 2013)

2. C. Eparu, R. Rădulescu, D. Stoica, Ovidius University Annals of Chemistry, 2, 83 (2013)

3. S. Neacsu, S. Suditu, C. Popescu, EDU '10 (Iwate Prefectural University, Japan, 2010)

4. C. Eparu, S. Neacşu, D. Stoica, Proceedings of the $21^{\text {st }}$ International Business Information Management Association (IBIMA) Conference (Viena, 2013)

5. A.J. Osiadacz, Simulation and Analysis of Gas Networks (Gulf Publishing, 1987) 